\title{
Effect of Laser Scribing on Soft Magnetic Properties of Conventional Grain-Oriented Silicon Steel
}

\author{
I. Petryshynets*, V. Puchý, F. KováČ And M. ŠEbek \\ Division of Metals Systems, Institute of Materials Research, Watsonova 47, 04101 Košice, Slovakia

\begin{abstract}
The present work investigates the influence of laser scribing of grain-oriented silicon steel on the improvement of its soft magnetic properties via magnetic domains modification. The experimental material was represented by conventional $\mathrm{Fe}-3 \% \mathrm{Si}$ grain-oriented electrical steel that was taken directly from industrial line after the final box annealing. The laser scribing treatments were applied onto the material surface in order to induce thermal stresses having crucial influence on the magnetic domains refinement. The laser scribing conditions have been optimized by introducing thermal stresses onto the experimental material surface leading to optimal refinement of final domains structures and desired magnetic softness minimization. A semiquantitative relationship has been found between the domain patterns and the used fiber laser treating method. The obtained modified domain structure led to a
\end{abstract} \\ significant decrease in coercivity measured in DC magnetic field from $20 \mathrm{~A} / \mathrm{m}$ to $12 \mathrm{~A} / \mathrm{m}$.
}

DOI: $10.12693 /$ APhysPolA.131.777

PACS/topics: 61.72.-y, 79.20.Eb, 68.55.jm, 77.80.Dj, 75.50.Vv

\section{Introduction}

Grain-oriented (GO) electrical steel is a soft magnetic material that is usually used in transformer core. Its magnetic properties are closely related to the sharpness of $\{110\}\langle 001\rangle$ crystallographic texture, i.e. the Goss texture, which is evolved by secondary recrystallization during the abnormal grain growth. It is the only product manufactured in the steel industry that applies the secondary recrystallization phenomenon [1].

The method of GO silicon steel production was invented by Goss in the 1930's and led to revolutionary reductions in core loss for $3 \%$ silicon-iron largely through the development of preferred orientation via the process of secondary recrystallization. Later, the procedure was improved, developed, and industrialized by ARMCO in the 1940's [2, 3].

Over the past 70 years, magnetic properties of grainoriented electrical steel have been improved dramatically. This has been achieved mainly by three technologies: (1) improvement of $\{110\}\langle 001\rangle$ alignment, (2) development of thinner-gauge material, and (3) establishment of magnetic domain refining techniques [4]. Magnetic domain structures of secondary recrystallized grains and especially their movement have a strong effect on the core loss of GO silicon steel. Pinning of domain walls movement and surface closure domains must be considered in order to reduce the magnetic losses of GO steel.

Several different domain refinement techniques, such as mechanical scratching, plasma irradiation, spark ablation and laser scribing have been studied for many years. All these methods can induce favorable stress that refines the magnetic domains due to mechanical or thermal strains introduction. For the industrial utilization of refinement process, the socalled "laser scribing" technique is usually applied due to its no-contact nature, the high flexibility and the little damage of the surface coating [5].

Typical core loss improvement of about $10 \%$ could be achieved with that kind of laser beam sources. The theoretically

*corresponding author; e-mail: ipetryshynets@saske.sk predicted core loss improvement of $70 \%$ indicates the motivation for further developments of the refinement process [6].

The objective of this work is to optimize the scribing process by means of fiber laser in order to reduce core losses of investigated GO steel by introducing favorable stresses leading to the magnetic domains refinement.

\section{Experimental procedure}

The material investigated in this work was the grainoriented steel with the following chemical composition, by wt\%: $\mathrm{C}=0.005, \mathrm{Mn}=0.190, \mathrm{Si}=3.100, \mathrm{P}=0.007$, $\mathrm{Al}=0.011, \mathrm{Cu}=0.45, \mathrm{~N}=0.010$. Strips of $0.35 \mathrm{~mm}$ thickness were taken after final box annealing from an industrial line. Before the realization of laser scribing, the investigated steel samples were prepared by electric spark cutting into small strips with dimensions $3 \mathrm{~cm} \times 1 \mathrm{~cm}$. The longest side of samples was parallel to the rolling direction. Then these strips were subjected to stress relief annealing at $800{ }^{\circ} \mathrm{C}$ for $30 \mathrm{~min}$ in dry hydrogen atmosphere.

The laser scribing process was realized on the surface of experimental samples by means of fiber laser with wavelength $1064 \mathrm{~nm}$ from TRUMPF company. The samples were treated in air by laser beam in continuous mode. The power density of the laser beam was in the range from $12 \mathrm{~W}$ up to $48 \mathrm{~W}$. During the application of scribing process, the laser beam spot on the samples surface with $50 \mu \mathrm{m}$ in size moved with the velocity of $100 \mathrm{~mm} / \mathrm{s}$. The laser scribing was performed in form of lines on the steels surface perpendicularly to the rolling direction. The distance between the lines was selected as following: $3,5,7.5$, and $10 \mathrm{~mm}$.

Soft magnetic properties represented by coercivity (magnetic softness) were measured before and after the laser scribing process on the each sample in DC magnetic field by commercial "Oersted type" coercivity meter KPS-lC.

The magnetic domains were observed by means of the Bitter method which is based on the use magnetic colloid suspension Ferrofluid for magnetic domains visualization in the light optical microscope "Olympus Inverted System Metallurgical Microscopy GX-71".

The most representative samples were chosen for the texture analysis which was carried out by an electron backscattered diffraction (EBSD) method. The data obtained were processed by the CHANNEL-5 software package. 


\section{Result and discussion}

The outgoing microstructure of the experimental GO steel which was taken from industrial line after final box annealing is presented by inverse pole figure (IPF) map in Fig. 1. Here, the EBSD analysis was carried out on sample surface perpendicular to its transverse direction (TD) and rolling direction ( $\mathrm{RD})$. As one can see, the material is characterized by primary recrystallized grain matrix with complete abnormal growing grains. The average grain size is approximately some millimeters in size. The IPF map shows that the presented grain matrix is formed by the grains with preferable $\{110\}\langle 001\rangle$ Goss crystallographic orientation. It means that axes of easy magnetization $\langle 001\rangle$ are parallel to the RD of sample.

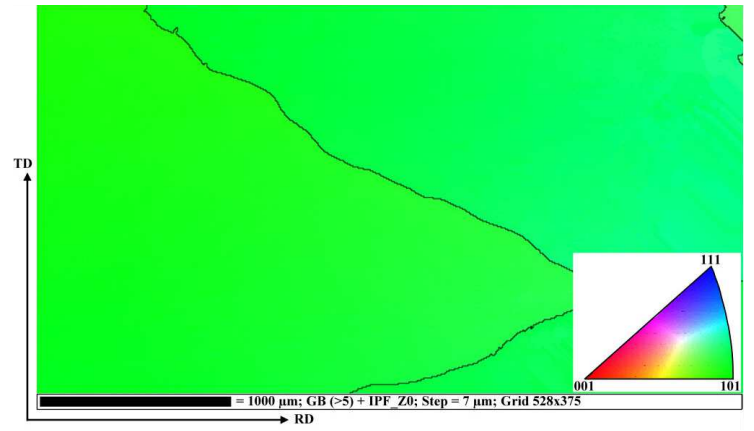

Fig. 1. The IPF map represents the microstructure and texture of experimental GO steel which was taken from the industrial line after final box annealing. Key for the identification of crystallographic orientation of grain is located in the bottom right corner of the figure.
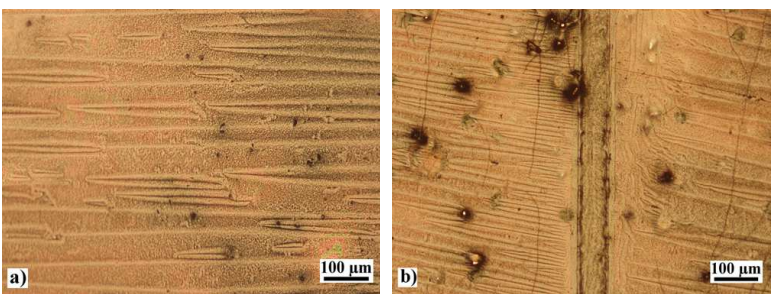

Fig. 2. 2. The domain structure of investigated GO steel: (a) without laser scribing and (b) after laser treatment by laser beam in continuous mode with power of $24 \mathrm{~W}$.

Figure $2 \mathrm{a}$ and $\mathrm{b}$ shows the magnetic domain structure of the sample before and after laser scribing where the image has been obtained by using the Bitter technique. This method based on the use of the colloidal suspension of iron oxide particles which are attracted by the external magnetic field and outlines the domain walls on the sample surface. Here, large $180^{\circ}$ magnetic domain walls oriented along the rolling direction are observed in the initial state, see Fig. 2a. As one can see, the width of domain structures is in the range $10-50 \mu \mathrm{m}$. The refined domain structure of samples which was induced by continuous laser beam is presented in Fig. 2b. Complex domain structures are observed in the vicinity of laser scribed lines (see Fig. 2b), wherein it can be assumed that degradation of domain walls takes place around the fastheated zones. The refinement of the magnetic domain structure after laser scribing is clearly visible, so it can be assumed that the eddy current component of the total losses will be lower after laser treatment.
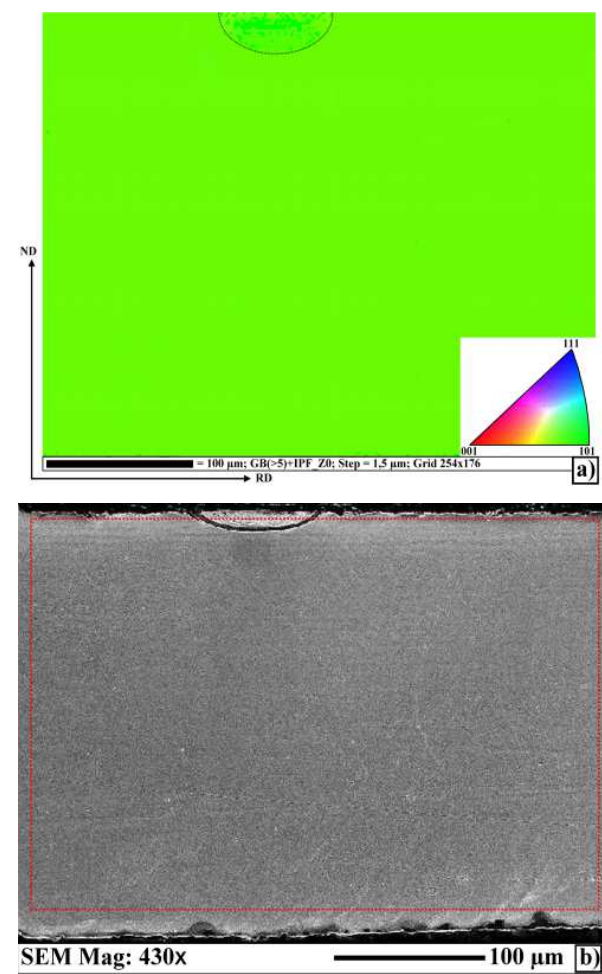

Fig. 3. (a) IPF map showing the crystallographic orientation of grain with heat affected zone (marked by black dashed line) obtained after scribing by continuous $24 \mathrm{~W}$ laser beam, (b) SEM microstructure of sample cross-section on which was carried out IPF map.
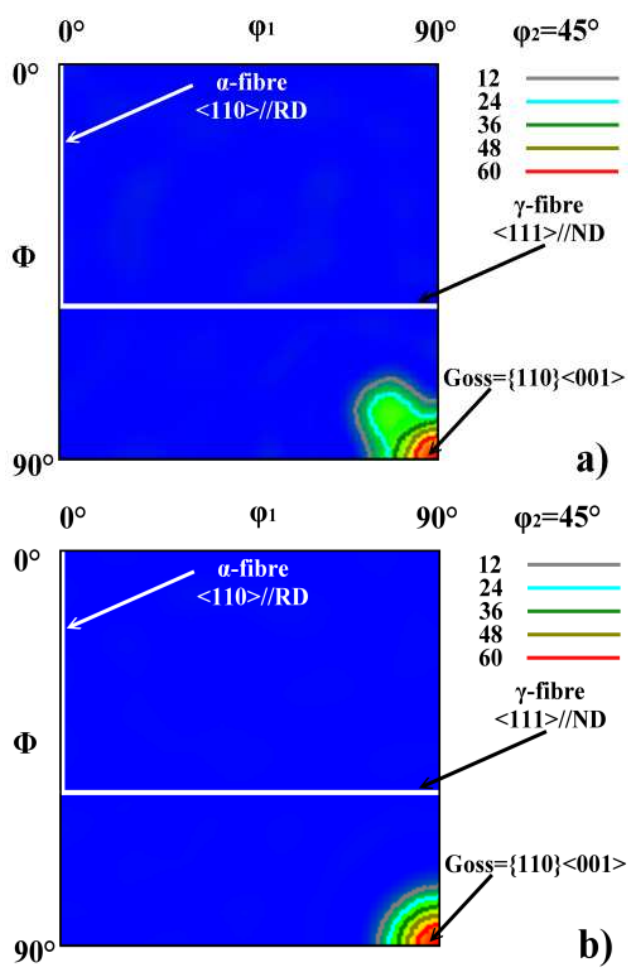

Fig. 4. The ODF sections at $\varphi_{2}=45^{\circ}$ represent the sharpness of Goss texture $\{110\}\langle 001\rangle$ for sample: (a) taken from industrial line after final box annealing and (b) after treatment by laser beam with power of $24 \mathrm{~W}$. 
The IPF map and SEM image of sample cross-section microstructure with the thermal affected zone which was induced by laser beam with power of $24 \mathrm{~W}$ is presented in Fig. 3a and $\mathrm{b}$, respectively. Here it is evident that laser affected zone is characterized by some substructural changes which are perfectly detected by SEM method, see Fig. 3b. On the other hand, the IPF map presents the part of grain affected by laser beam (marked by black dashed line) which is characterized by the Goss texture. The comparison of the Goss crystallographic texture sharpness measured in the samples before and after laser beam treatment with power of $24 \mathrm{~W}$ is shown in Fig. 4a and b, respectively. It is clearly visible that the laser scribing does not have a strong influence on the sharpness of the Goss texture. This indicates that laser beam with power of $24 \mathrm{~W}$ interacting with the steel surface induces only slight changes of substructure parameters in the place of contact and the texture of the sample before and after laser scribing is similar and characterized by high intensity of $\{110\}\langle 001\rangle$ Goss component.

The magnetic softness (coercivity) change of the samples after application of laser scribing process is presented in Fig. 5. Here, the coercivity change $\Delta H_{c}$ is the mathematical difference between the coercivity measured in the samples before and after laser scribing. Because of this the negative values of $\Delta H_{c}$ describe the deterioration of magnetic softness of laser treated samples. As one can see, the samples which were scribed by laser beam with the distance between lines of $3 \mathrm{~mm}$ are characterized by deterioration of coercivity. It could be explained by high intensity of thermal stresses on the samples surface. The improvement of coercivity were achieved for the GO steel samples which were laser treated with distance between lines of $5,7.5$, and $10 \mathrm{~mm}$, respectively, see Fig. 5 . Here, the lowest coercivity value was measured in the sample which was subjected to laser scribing with line distance $7.5 \mathrm{~mm}$ and power laser beam of $24 \mathrm{~W}$. The obtained samples with modified domain structure exhibited significant decrease in coercivity measured in DC magnetic field from $20 \mathrm{~A} / \mathrm{m}$ to $12 \mathrm{~A} / \mathrm{m}$.

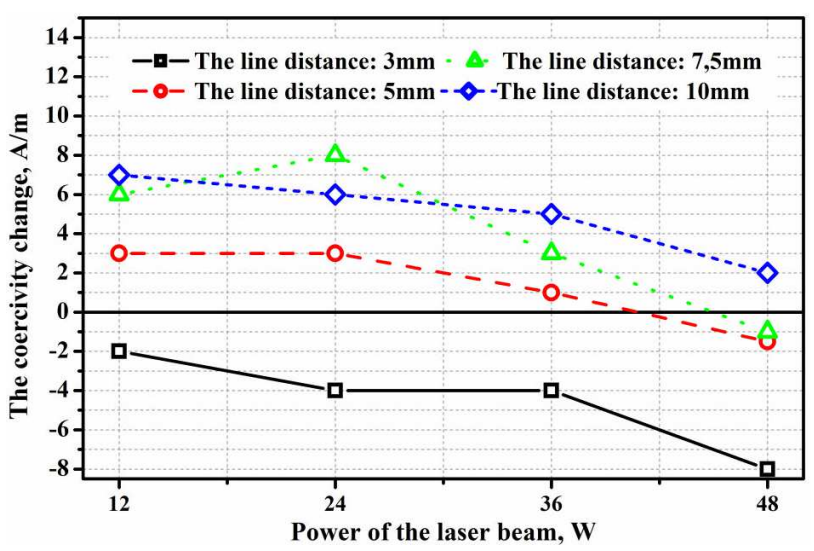

Fig. 5. Dependence of the coercivity change $\Delta H_{c}$ on laser beam power and distance between the scribing lines.

The improvement of soft magnetic properties of GO silicon steels subjected to laser scribing is mainly attributed to magnetic domain refinement. These kinds of steels are characterized by microstructure with huge grain size resulting in the enlargement of magnetic domains. These large domains structures increase magnetic losses as the domain walls move back and forth under the action of alternating field. If the domain size is kept small, the walls do not move as far and less energy is lost in moving them. The laser scribing enables to reduce the average size of magnetic domain walls because during this process the laser beam introduces thermal stresses on the surface of the material. This thermal input is localized to a small spot on the surface.

Because the surrounding material is not affected, the heated area is constrained to react only by expanding out of the plane of the material surface. This rapid surface displacement launches a dilatational wave that propagates the stress distribution throughout the depth of the material. The resultant strain is believed to cause a slip in the plane that provides the new wall boundaries [6].

\section{Conclusions}

The magnetic domains refinement was performed by continuous laser beam depending on its power density and line distances on the samples surface. The experimental results have clearly shown the beneficial effect of laser scribing on the improvement of the magnetic softness of GO steels. The energy of laser beam induces the thermal shock which in combination with correct density of scribing lines can decrease the samples coercivity. The texture analysis demonstrated that small spots of thermal stresses do not influence the outgoing crystallographic orientation. Moreover, the measurements on the samples treated at optimal conditions show a significant reduction of coercivity from original $20 \mathrm{~A} / \mathrm{m}$ to newly obtained $12 \mathrm{~A} / \mathrm{m}$.

\section{Acknowledgments}

This work was carried out within the project, which is supported by the Slovak Research and Development Agency under the contract No. APVV-15-0259. This work was also partially supported by the Slovak Grant Agency VEGA, project No. 2/0081/16, No. 2/0120/15 as well as COST Action MP1401. Also, the work was realized within the frame of the project ITMS 26220220037, ITMS 26220220061, and ITMS 26220220064 .

\section{References}

[1] W. Guo, W. Mao, J. Mater. Sci. Technol. 26, 759 (2010).

[2] T. Kubota, M. Fujikura, Y. Ushigami, J. Magn. Magn. Mater. 215-216, 69 (2000).

[3] A. Morawiec, Scr. Mater. 64, 466 (2011).

[4] Z. Xia, Y. Kang, Q. Wang, J. Magn. Magn. Mater. 320, 3229 (2008).

[5] P. Rauscher, J. Hauptmann, E. Beyer, Phys. Proced. 41, 312 (2013).

[6] S.V. Ponnaluri, R. Cherukuri, P.A. Molian, J. Mater. Process. Technol. 112, 199 (2001). 